\title{
Extension and measurement: A constructivist program from Leibniz to Grassmann
}

\author{
Erik C. Banks \\ Department of Philosophy, Wright State University, 3640 Colonel Glenn Hwy, Dayton, OH 45435, USA
}

\section{A R T I C L E I N F O}

\section{Article history:}

Received 16 February 2012

Received in revised form 22 June 2012

\section{Keywords:}

Leibniz

Grassmann

Riemann

Extension

Algebra

Space

\begin{abstract}
A B S T R A C T
This paper traces what I see as a Leibniz-inspired constructivist program through the eyes of the 19th century philosopher-mathematicians Herbart, Riemann and Grassmann, and then uses Grassmann's algebra of points to build up levels of extension algebraically. The connection between extension and measurement is investigated in line with this constructivist program.
\end{abstract}

(c) 2012 Elsevier Ltd. All rights reserved.

When citing this paper, please use the full journal title Studies in History and Philosophy of Science

\section{Introduction}

Extension is probably the most general natural property known. But is it a simple, fundamental property or is it constructed out of still more fundamental properties or relations? Descartes claimed, along with most philosophers and scientists, that extension was a simple property that could neither be explained nor constructed. Leibniz denied that extension was a fundamental property, and followers of Leibniz, such as Herbart, sought an explicit construction of extension from unextended points and forces in a physical monadology. Finally the mathematicians Riemann and Grassmann took a turn at developing extension from scratch, without assuming the property as fundamental. I will trace this constructivist line of argument to Grassmann's algebra of extension and show how to interpret it in this light. Constructivism is a minority view, but its story is worth telling, for, despite the magnitude of the names involved in some way with this tradition (Leibniz, Herbart, Riemann and Grassmann), the problem of constructing extension has attracted very little interest among philosophers or historians and is long overdue for a taking stock.

\section{Extension: Concept or Intuition?}

The first problem is to define extension. What definitions there are, from the medievals to Kant, talk about the apartness of parts of magnitudes like a length or a time, the parts of an object, stages of a process and so forth. The opposite is an intensive magnitude with a degree or intensity, but no extended parts. These definitions are clearly circular, since 'apartness' assumes the intuition we are trying to define. Also, a length and a degree, say of temperature or density, are both representable in the same way by the real numbers $\mathcal{R}$. At no point is any appeal made to the outside-ness or inside-ness of parts or degrees. The intuition of extension plays no role in the mathematical construction of the reals; it rather has to do with how we represent the real continuum. Second, the distinction between discrete and continuous magnitude plays no role here either, since extensions can be either discrete or continuous.

Generally speaking, mathematics makes no distinction whatever between an extended representation, say of the real numbers, and any other interpretation. You could think of sets as points collected in extended space, or as anything else you wish. Nowhere in

E-mail address: erik.banks@wright.edu 
mathematics is it ever specified what would make for an extended interpretation. When a specific spacetime structure is built up, we assume a blob-like extension of points, call it $\mathcal{R} \times \mathcal{R} \times \mathcal{R}=\mathcal{R}^{3}$. Then we define a topology for the blob (an open ball, a sphere with boundary, a torus) by laying constraints on neighborhoods around points. A coordinate mesh can be imposed, the curvature and the metric can be defined, all the way up to the causal structure of lightcones in the space, but nowhere has the assumption of the extended blob been justified as opposed to any other interpretation of the sets making up $\mathcal{R}^{3}$. We are forced to conclude that extension, just like sense or order, ${ }^{1}$ is not defined in mathematics, rather it is an intuition we bring to mathematics when we make an extended representation of its abstract structure.

This brings us to Kant's view, that the extended 'drafting board' of intuition, on which all extended constructions are done, is necessarily assumed and cannot be analyzed, or brought under concepts, because any conceptual analysis would assume extension and beg the question. Assuming one tried to generate a dynamical construction of space from causal interactions of spaceless, timeless entities like monads, it seems one would be bound to assume a causal interaction over space and time between the monads, so that the construction must fail. It has even been proposed that all dynamical, causal derivations of space and time are doomed to failure, even in advanced physics, since dynamics and causation simply assume extended length. If this were true, Kant would be right to call extension an intuition we bring to all extended representations of the world, rather than a concept, for apparently we have no conceptual grasp of it at all. ${ }^{2}$

\section{Evidence for a Leibniz-Inspired Constructivist Program}

A philosophico-scientific program for a construction of extension certainly existed in Germany, at least up until the time of J.F. Herbart, Riemann and Grassmann. The construction originally took the form of what was called a physical monadology, inspired by Leibniz's writings, and pursued by Christian Wolff, Roger Boscovich and the Pre-Critical Kant among others (Kant 2003; Friedman 1998). As we shall see in more detail below, Herbart also developed such a physical monadology in his Allgemeine Metaphysik of 182829 , which involved unextended point-like Wesen (beings, entities) and instantaneous forces, and where extension is traced out by an associative-dissociative diffusion of forces. Herbart, in turn, is a direct historical link to the German mathematician Riemann (Banks 2005; Scholz 1982) and is even mentioned by name alongside Gauss in Riemann's seminal 1854 Probevorlesung on the foundations of geometry. Grassmann looked back to Leibniz and claimed that that thegeometric algebra developed in his groundbreaking 1844 Ausdehnungslehre was the natural development of Leibniz's ideas on the "geometric characteristic," announced in the letter to Huygens in 1679. Grassmann's prize essay of 1847 was written in response to a challenge to complete Leibniz's project for the characteristic. ${ }^{3}$ Grassmann claimed that because his geometric algebra described abstract entities and relations at a level prior to extension, they could be used to analyze the concept of extension, where sensory human visualizations could no longer reach. Grassmann, writing about himself in the third person, also attributed this goal to Leibniz:
Finally, at the end of Leibniz's presentation [the 1679 letter] is yet another remarkable point where he quite clearly expresses the applicability of this analysis to objects that are not of a spatial nature, but adds that it is not possible to give a clear concept of this in a few words. Now, in fact, as is demonstrated throughout Grassmann's Ausdehnungslehre, all concepts and laws of the new analysis can be developed completely independently of spatial intuitions, since they can be tied to the abstract concept of a continuous transformation; and, once one has grasped this idea of a pure, conceptually interpreted continuous transformation, it is easy to see that the laws developed in this essay are also capable of this interpretation, stripped of spatial intuitions. (Grassmann 1995, p. 384)

\section{Herbart: A Transitional Figure}

J.F. Herbart (1776-1841) was Professor at Königsberg and Göttingen. He was famous for his mathematical psychology of psychic forces and for his philosophy of spatial representation. Herbart was a realistic, scientifically minded philosopher who advocated constructivist methods and who challenged the Kantian a priori drafting-board intuition of space and time extension, believing he could analyze it further. Herbart is often considered a Leibnizian for this reason, because he worked on constructing space in a physical monadology, like Christian Wolff and the young Kant before him. In his Metaphysik, Herbart describes point-like Wesen possessed of instantaneous forces. He then describes associative-dissociative extension-tracing processes among the Wesen that amount to a construction of extension. Herbart also believed, as Leibniz had, that the primary forces of nature, at this metaphysical level, were instantaneous and did not require an extended representation. What could he have meant by this? Of course if we simply take the notion of force straight from mechanics it is not true: a freely acting force always requires some space to act through (producing energy) and some time to act over (producing momentum) and manifests in accelerations. However, as the natural philosopher and metaphysician Roger Boscovich pointed out (1966), we can imagine that there are no free forces at the primary level, but instead that each primary 'active' force always acts against a countervailing 'passive' force. Boscovich proposes that the greatest active force is equilibrated by the smallest passive force in a small enough 'snapshot' of time and space. Actually there are two possibilities: 1 ) either the stronger force will always overcome the weaker continuously and there never is a minimum, or else 2) there are discrete extended quanta of energy-momentum which cannot be made smaller, even when the greatest force acts against the weakest. Beneath that extended level, all is in stasis: we can only assemble extended quanta out of static, combinatorial patterns of primary forces in equilibrium.

Let us suppose this balance point to have been reached. Herbart now sees two kinds of instantaneous relationships for the primary forces: they either depend on each another (Zusammen), or they do not (Nicht-Zusammen). When the forces are put into a static combinatorial pattern, their instantaneous relationships, which we express in symbols, can bear the intuitive interpretation of the serial tracing of an extension, see Figure 1 below.

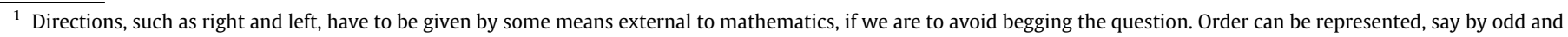

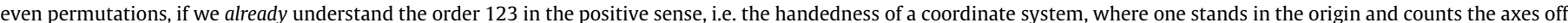

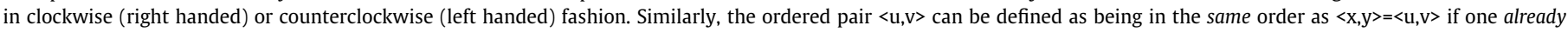
understands what the order of $\langle\mathrm{x}, \mathrm{y}\rangle$ is. Russell (1903) proposes including order or sense among the undefined notions, but says nothing about extension.

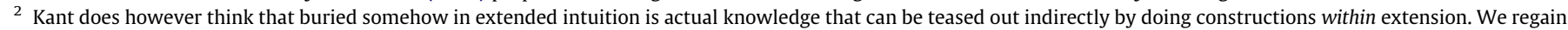
explicitly what we have already read-in through intuition.

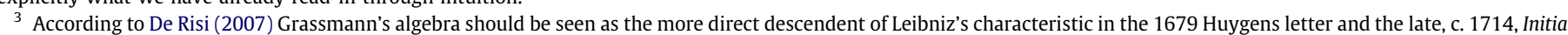

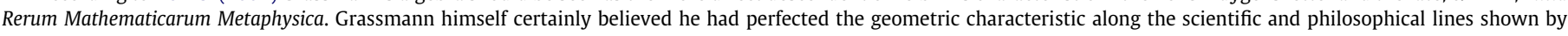
Leibniz.
} 


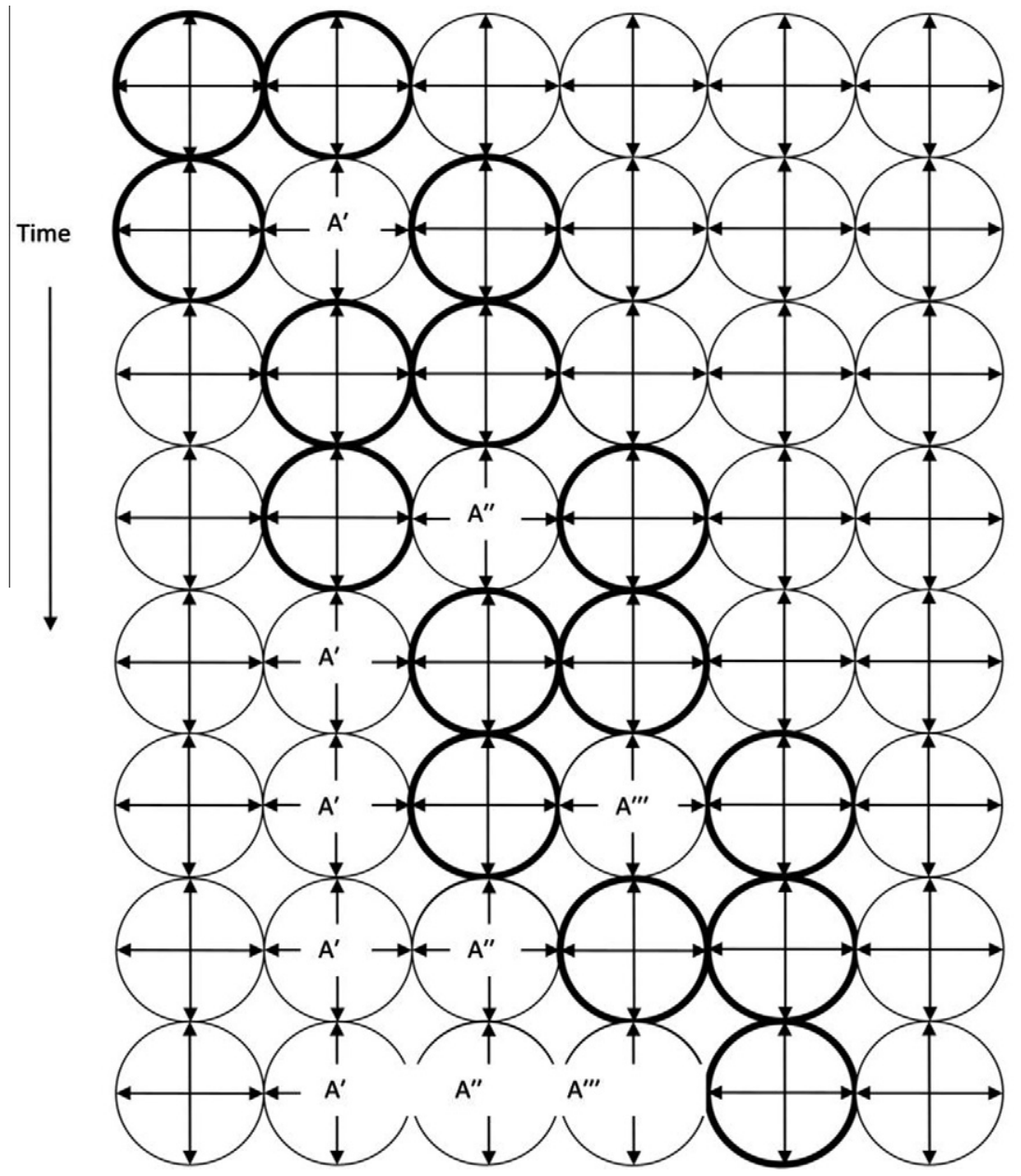

$B+A$

A-B

$B+A$

A-B

$B+A$

A-B

$B+A$

Figure 1. Herbart's tracing process.

In his Allgemeine Metaphysik (Herbart 1964 §245, 2005) Herbart imagines such an 'inchworm' process, tracing out a line of discrete points and dissociating gaps. Let $A$ and $B$ be the names of the static nuclei that do the tracing. $(A+B)$ represents the operation of associating B to a fixed A, (A-B) represents "taking B away" from fixed A, dissociating $B$ from $A$. This gives us an infinite symbolic sequence:

$(\mathrm{B}+\mathrm{A})+(\mathrm{A}-\mathrm{B})+(\mathrm{B}+\mathrm{A})+(\mathrm{A}-\mathrm{B})+(\mathrm{B}+\mathrm{A}) \ldots$

We also need an operation to represent what Herbart calls the generation of images. Sticking with the intuitive interpretation, when the process moves on we need a way to represent its past stages. That is, we need some other present nucleus to serve as a recordor copy of where A has previously been, called A', A", A"' and so forth. The copies are generated, in sequence, by the number of times we dissociate B from A and reassociate A with B. Finally, we can express the order of the series of associations and dissociations by bracketing or nesting the previous stages inside the later stages (which I have omitted above).

Herbart's generation of a row of dots is an attempt to analyze the intuitive content of extension through concepts or symbolic operations. Strikingly, so far as I know, he is the first to recognize in extension the need for an alternation between a dissociating operation, where his instantaneous forces are independent of each other, as well as an association, where the forces are dependent. We need not, and should not, think of these operations as involving motion, but rather through a more abstract concept of constancy and variation, as might be true of two functions A,B: A is capable of variation, while B holds constant.

Herbart insists that his symbolic processes are adequate serial generations and yet are not to be visualized in intuition. This must have been a common objection which he strains to answer in many places in his work. In his defense, the symbolic processes are interpretable as the serial generation of points, like a tracing, but the symbolism also bears all possible interpretations and not just as the intuitive serial process. Herbart's 'inchworm' process strikes me as a primitive sort of program, automating a series of steps and operations, but which is also present as a complete object, indifferent to serial order of any kind. Any stage of the process could be represented as the present one, the centered, present perspective of a particular track through space, by altering the order of nesting. The process can also be reversed in direction by pushing through a minus sign, giving us a row of $-A$ 's, or, in keeping with our conventions a row of B's (suggesting that $A=-B$ and $A+B=0$ ). We require only:

1. Associative and dissociative operations in an alternating pattern,

2. A copy operation which reproduces past stages, or identifies other present stages as records,

3. Universality: any stage can be considered present stage of nesting, any image can be made the present relative to other stages, any point can be the present center of perspectives of a particular track through the space. 
Herbart also provided an analysis of extension in psychology, for extended sensory manifolds of sight, color, sound and touch. These manifolds are built up point by point through processes he called reproduction series (Banks 2005; Boring 1950). For example, an individual colored point does not actually appear in a three dimensional color-space given in advance, with all the points filled in around it. Instead Herbart imagined an individual point changing in hue as it 'moves' around the manifold on its own track. The previous stages of the process are reproduced in memory and the memory images are added, in associated-dissociated order or a combinatorial pattern preserved in memory, to the present stage of the point. Every such path traced in the manifold makes up an individualized perspective in which colored points are encountered in a series, from within. There is no outside view; the manifold is just the sum total made up of views along all these separate individual tracks.

The changes of a colored point, or the tracing out of a row of dots in the Metaphysik, do indeed make up an extension, but this extension is intuited only in the memory or records of non-present images. The present stage is always an unextended point. Herbart then claimed that an actual intuitible extension of a length required two reproduction series, crossed over each another. When two reproduction series are crossed over, or associated and dissociated with each other, this more complexly structured process appears in an intuitable way, as a length made up of separated points. I call this the wire argument, for short. Consider a wire seen headon as a point. The extension of the wire is not intuitable unless the wire is turned through some independent direction, associated with the first, but also allowing us to dissociate the points on the wire from each other. Two reproduction series do that in a causal sense. From within the manifold, running along a track, one traces out and reproduces the points visited not in one direction but in two. In Figure 2 below, imagine one series A,B,C,D,E with each letter potentially associated with any of the range of letters run through in the other series $\mathrm{V}, \mathrm{W}, \mathrm{X}, \mathrm{Y}, \mathrm{Z}$. As we pass from $\mathrm{A}$ to $\mathrm{B}$, we can imagine traversing the whole range of the other series, setting them apart. Nevetheless the closeness, and the order of the association between $A$ and $B$ is preserved, since no particular associations are created between $A$ and any individual letter of the other series.

The difficulty in seeing such an analytical construction as actually extended in intuition was remarked upon by psychologists, including William James, and by Gary Hatfield in his comprehensive study of spatial representation in psychology (1991). I think this was because Herbart's readers expected to stand outside the whole space and observe its extension from the outside like a

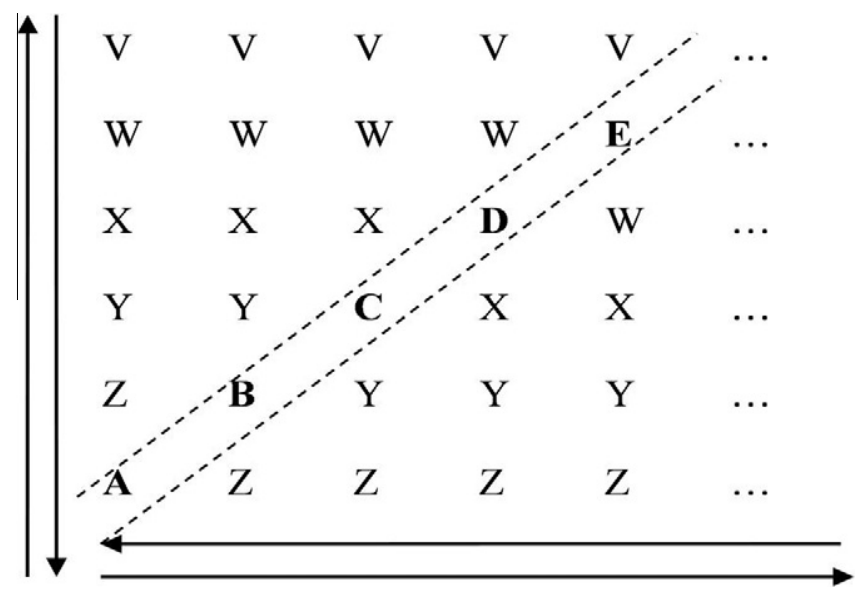

Figure 2. Herbart's reproduction series. box, whereas Herbart had always insisted that the extension of space only appears from within, where the point of observation is always an internal one from an origin of perspective to other points encountered on other tracks. Mathematicians sometimes call this an 'intrinsic' view of space, thinking of Gauss who developed a theory of curvature that lies within the surface studied, instead of the ambient space. This desire to 'see in from outside' harkens us back to the science museum fallacy, say of representing the entire universe, while we seemingly stand outside in nowhere. From Herbart's point of view, all realistic spatial representation is intrinsic. We have no idea what space would look like from outside space and it is a metaphysical error to think we could.

\section{Riemann on Multiply Extended Magnitude}

Bernhard Riemann discusses the problem of what he calls multiply extended magnitude in his famous lecture "On the Hypotheses that Lie at the Foundation of Geometry" (1854, 1954). He credits Herbart by name, alongside Gauss no less, and mentions some philosophical investigations that helped him. Most scholars now think that Herbart's psychology of sense manifolds of color and sound and the crossed reproduction series had a greater impact on Riemann than the construction of points in the metaphysics (Russell 1996, orig. 1897; Scholz 1982). Riemann takes color space and (in notes for the lecture) the tone row (see Banks 2005; Scholz 1982) as illustrations of three and one dimensional manifolds. He also says right away that he does not intend to assume the intuitive drafting board of space on which to do constructions, but that he intends to derive the concept of multiply extended magnitude from general concepts of quantity. The first task is to define a manifold extension. The second task is to give definitions of intrinsic curvature and measure determined from within this extension, say by introducing rigid meter sticks or light beams.

The first task is the one which most interests us here (see also Banks 2005 for a more detailed view of Riemann's ideas about space and especially their connection to his natural philosophy). A manifold, Riemann says, consists of various modes of determination (Bestimmungsweisen), a minimum of two. The points and elements of the manifold are determined by transitions, discrete or continuous, between these modes.

Notions of quantity are possible only where there exists already a general concept which allows of various modes of determination. According as there is or is not found among these modes of determination a continuous transition from one to the other, they form a continuous or a discrete manifold; the individual modes are called in the first case points, in the second elements of the manifold (Riemann, 1953).

In the color manifold, the modes of determination are clearly qualities, colors. As the colored point changes, its mixture of color qualities exchange continuously and different colored points are thereby determined. Similarly, as the tone slides through high and low along the tone row, it exchanges directions of forward and backward, like a point on a one dimensional line. What determines the structure of the manifold is the number of these possible qualities, the directions of the manifold, and the properties of the transitions. For example the two dimensional line depends on its two directions, forward and back, and a continuous transition:

In a concept whose various modes of determination form a continuous manifold, if one passes in a definite way from one mode of determination to another, the modes of determination traversed constitute a simply extended manifold and its essential mark is this, that in it progress is possible from any point only in two directions forward or backward (Ibid.). 
Riemann also indicates that predicates or class concepts can serve as modes of determination in the most general manifolds by which individuals may simply be classed together, identified and counted if they are discrete objects. Why must we have at least two such modes of determination? Probably because if modes were predicates, then individuals simply fall under them or they don't. We want to be able to further differentiate individuals all alike in some property. This we can do by introducing some other predicate to set them apart. All men of a certain height can be separated by weight. A third predicate, wealth, can separate them further. Riemann thus generalized the idea of a manifold as far as it would go into the abstract. You can have manifolds of directions, qualities, or physical properties, their structure defined by their number, dimension, and the nature of the transition, discrete or continuous. These properties can be independent, which is required whenever we want to differentiate an individual from another. But the properties are also clearly dependent at points or individuals determined by the transitions. It would thus seem that the modes are capable of dependence in individuals, and independence in the interstices, and that the transition combines both the dependence and independence of the modes, alternating as they transition from one individual to another.

Turning to the question of measure, Riemann says that in the case of a discrete manifold, the definition of measure is built into the manifold extension itself. We have only to count up the points traversed as the modes switch discretely in their values. Riemann then says that measure can only be defined for a continuous manifold by selecting an external standard of measure, like meter sticks and light rays. He restricted this condition further by demanding that the space exhibit constant curvature, line elements becoming little arcs, a requirement we do not retain today as we accept spaces of non-constant curvature caused by matter and energy in the theory of relativity.

However, as Howard Stein has pointed out (1977), Riemann may have left an important door open by referring to standards of measure approaching the "infinitely small" where the physical meter stick and light ray are no longer applicable because of physical changes in those standards. The stick becomes atoms, the light ray becomes bent by refraction in matter. The famous phrase here is that new standards of measure could be sought in the "binding forces" that act on the manifold itself. Meaning, as also Stein seems to suggest, that just as in the discrete case, so too in the continuous case we may well find the properties of space internally welded into the manifold itself, and not in externally imposed standards of measure, but in these internal ones. This would support the interpretation I gave of manifold extension above, as a manifold of physical properties which could later be exploited again when we are searching for those physical standards of measure intrinsic to the manifold itself and useful in the infinitely small where other standards break down.The crucial passages in Stein supporting this reading seem to me to be:

...our spatial notions, insofar as they are brought to bear in physics, have their significance only as structural aspects of a more embracing structure: that of physical interaction itself. This is how I understand one most important remark of Riemann's that does seem to me obscurely phrased: his famous remark that if "the reality that lies at the basis of space" is not a discrete manifold, then "the ground of its measure-relations must be sought...in binding forces that act upon it. Setting aside the issue of discrete versus continuous the essential point seems to me to be this: By "the reality that underlies space" Rie- mann means that aspect of the real structure of the world which we express in terms of spatial concepts. (Stein, 1977, p. 24-25)

I am speculating here about what Riemann may have meant and what Stein may have meant in interpreting Riemann. But if I am reading them both correctly, these observations, and the commentary, would fit very well indeed with the idea of a manifold composed of physical properties at bottom, as its modes of determination.

\section{Hermann Grassmann's Ausdehnungslehre}

Many of the above ideas find a natural home in Hermann Grassmann's masterpiece, the Ausdehnungslehre of 1844, 1861 (Grassmann 1995; for illuminating studies of the system see Crowe 1967; Lewis 1975; Schubring 1996; Browne 2009; Petsche, 2011a,b). Grassmann's work is devoted to an analysis of the concept of extension in all generality, of which space forms only a special case. His chief innovation is the exterior product $(\wedge)$ an anti-commutative extension-building operation, which takes the geometric product of two magnitudes. Points $\alpha, \beta$ for example multiply to a directed line segment, $\alpha \wedge \beta$, which we call a (bound) vector, and vectors $A, B$ multiply to a paralleogram area element, $\mathrm{A} \wedge \mathrm{B}$ known as a bivector. This operation can be continued to trivectors and n-grade entities.

As Grassmann was aware, the geometric entities, and the basic set of algebraic operations on them, can be defined without the assumption of a coordinate system or a metric, and are all extremely basic, tensorial entities. They are thus well suited for interpretation as physically real entities, but also as general slots one can fill with a variety of entities: vectors, differential forms, operators, even transformations. When metric notions are introduced in Grassmann algebra, an orthogonal complement and inner product can be defined and the powerful Clifford algebra of rotations can be introduced, which incorporates Hamilton's quaternions, the complex numbers, and the algebra of matrices (see Browne 2009; Hestenes 1999; Doran and Lasenby, 2007). These ideas are currently being applied to give an intuitive geometric interpretation of quantum mechanics.

In the rush to geometricize, however, what has not received so much attention is that Grassmann, like Riemann and Herbart, insisted that his entities and products not be understood primarily as extended geometric entities in a background drafting board of space:

It is important to keep in mind that all of the elements so generated are not to be conceived as already given otherwise, as perhaps in the theory of space all points are originally given through the presupposed space, but rather as being generated from scratch (Grassmann 1995, p. 50).

Grassmann deliberately used an abstract philosophical language of 'systems,' 'evolutions,' and abstract 'constancy' and 'variation', to avoid assuming extended intuition. This language severly hampered the reception of the work by mathematicians, even by Hamilton, who was philosophically inclined (Crowe 1967). Grassmann could have left out the philosophy and stuck to the math, but it was clearly essential to what he thought he had achieved. We know he was a serious student of philosophy (Lewis, 1975) and he may well have known Herbart's works in some detail, perhaps through his friend and colleague Karl Scheibert, which seems likely (Petsche, 2011a,b). 


\section{Grassmann's Point Algebra and its Interpretation}

Let's begin at the simplest level with Grassmann's algebra of points and free vectors. ${ }^{4} \mathrm{~A}$ point is defined by an arbitrary zero point and a free vector which carries the origin to the point, $\mathbf{P}=\mathrm{P}_{0}+\mathbf{v}_{\mathrm{P}}$. Points are bound to their position. They also have a weight, aP, positive or negative, with 1 as the unit weight of a geometric point with position only, $1 \mathbf{P}=\mathbf{P}$. Points sum barycentrically to their center of gravity. Two positive, equally weighted points sum to a point of double the weight on the line between them. Two unequally weighted points sum by their respective weight-distances from their center of gravity, see Figure 3.

Two unit points, can also be subtracted, $\mathbf{P}_{2}-\mathbf{P}_{1}$, cancelling the origin and leaving a free vector from $\mathbf{P}_{1}$ to $\mathbf{P}_{2}$ : $\mathbf{P}_{2-}$ $\mathbf{P} 1=\left(\mathbf{P}_{0}+\mathbf{v}_{\mathrm{P} 2}\right)-\left(\mathbf{P}_{0}+\mathbf{v}_{\mathrm{p} 1}\right)=\mathbf{v}_{\mathrm{P} 2}-\mathbf{v}_{\mathrm{P} 1}$. This free vector is called a point at infinity and has only direction and no weight or location, see Figure 4.

A basis for level 1 is an arbitrary $\mathbf{P}_{0}$ and three free unit vectors $\mathbf{e}_{1}, \mathbf{e}_{2}, \mathbf{e}_{3}$. Grassmann himself separated the bound and the free entities into two different algebras, and some Bankss have followed. A combined interpretation (Swimmer 1996; Browne 2009), which will be followed here, allows bound entities and free entities to share the same algebra at the same dimension of level 1.

The two most common physical interpretations of the point calculus are Archimedes' law of the lever and the determination of the center of mass. It is also possible to interpret the point as the potential energy determined from an arbitrary zero. For example, imagine starting with a particle at the origin and imagine the vector as a transition, doing work by taking the point a weighted-distance from a force center. Grassmann also gave an interpretation of the point calculus for deriving his famous law of color mixtures (see his original diagram in Figure 5 below). This is a color wheel theory, approximately true of actual color vision, in which he represented white at the center $(\mathrm{O})$ and represented the hue of each color by its direction from $O$. The weight of the point represented the saturation or mixture of white to the pure hue at the rim.

Grassmann's law states that the colors add barycentrically just like weighted points. This has the consequence that every color will have a complementary color and that a mixture (of lights not pigments) will contain some added white (as A and B sum to $C$, not D). Clearly this is not an extended space which requires we actually fill it in with all of the colored points. It is a space of classification of points by their qualities, more abstractly defined, and similar to the general manifold of qualities or properties used by Riemann. We can continue further in this line by offering a philosophical interpretation of points, removing extension altogether, along with metric concepts of weight and length, and allowing the free vectors to be properties or predicates Fx, Gx, $\mathrm{Hx}$, leaving only a bare manifold of individuals classified by properties, see Figure 6 below. This is an affine space, where we need only preserve parallelism of directions, but where only a notion of relative position or length makes any sense, a point is some-

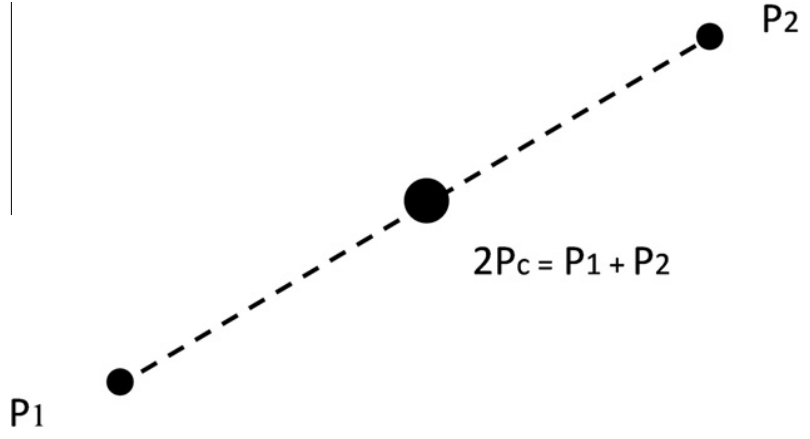

Figure 3. Barycentric point summation.

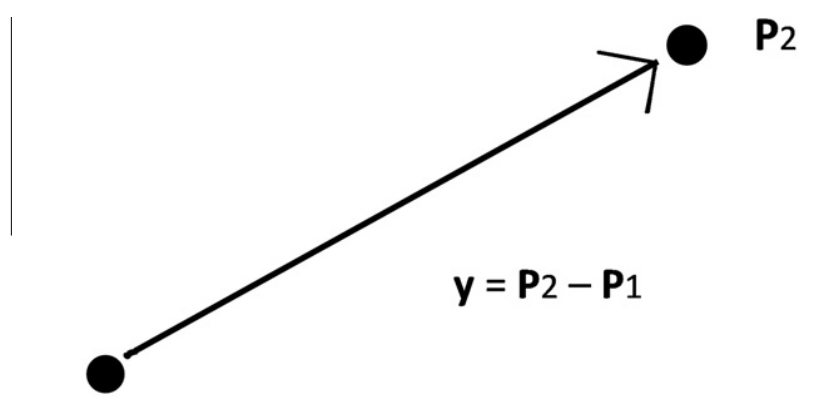

$\mathbf{P}_{1}$

Figure 4. Free vectors by point subtraction.

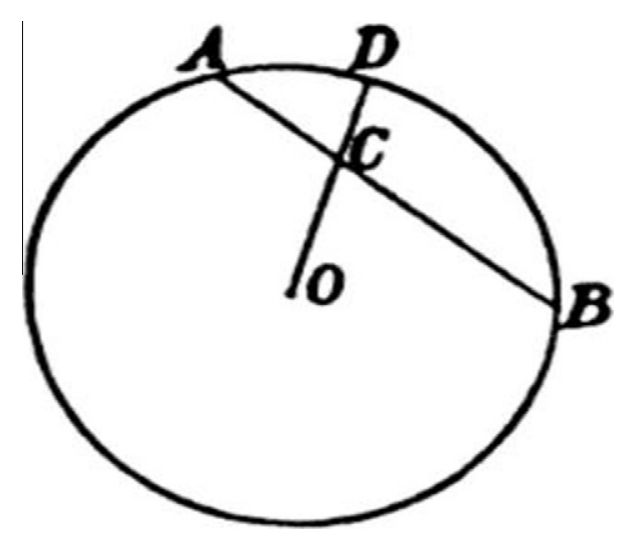

Figure 5. Grassmann's law of color mixtures.

where between others, a transition is determined by relative endpoints only. It is also not possible to sum points in affine space. ${ }^{5}$

Again, we can think of an individual as determined by a transition from an arbitrary zero point, but now, since the free vectors

\footnotetext{
${ }^{4}$ Following Swimmer (1996) we can characterize the points as follows. Let $\{A, B, C . .$.$\} be points, let A=[A]+\mathbf{v}_{\mathbf{A}}$. Meaning let it have a weight $[A]$ and a position vector $\mathbf{v}_{\mathbf{A}}$. If $B$ and A have equal weights, let B-A be the 0 weighted point, or free vector, $\mathbf{v}_{B}-\mathbf{v}_{A}=a\left(\mathbf{v}_{B}-\mathbf{v}_{A}\right)$. Let the free vectors of level one obey all of the usual rules of a vector space, addition, subtraction, multiplication by scalars, inverses and zero vector. Let the points obey the following rules for barycentric addition, subtraction, multiplication by scalars and additive inverses:

1. $A+B=B+A$

2. $A+(B+C)=(A+B)+C$

3. $\mathrm{A}+-\mathrm{A}=0$

4. $[\alpha A]=\alpha[A]$

5. $(\alpha+\beta) A=\alpha A+\beta A$

6. $\alpha(A+B)=\alpha A+\alpha B$

7. $\alpha(\beta A)=(\alpha \beta) A$

5 Thus weight like length of vectors must be considered a metric notion.
} 


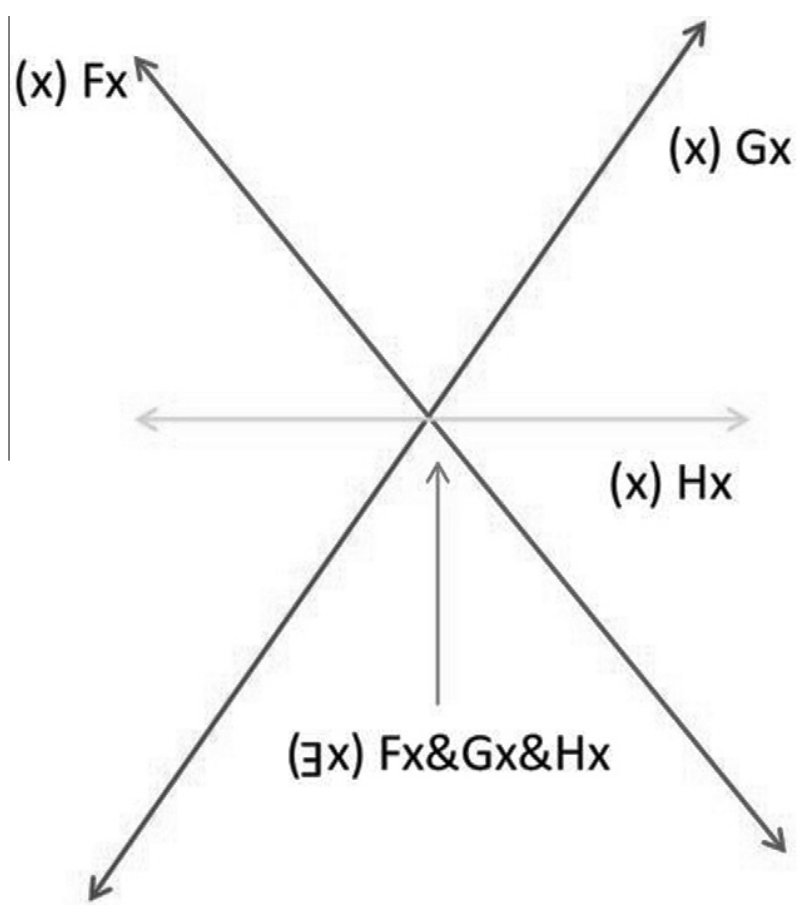

Figure 6. Abstract manifold of properties.

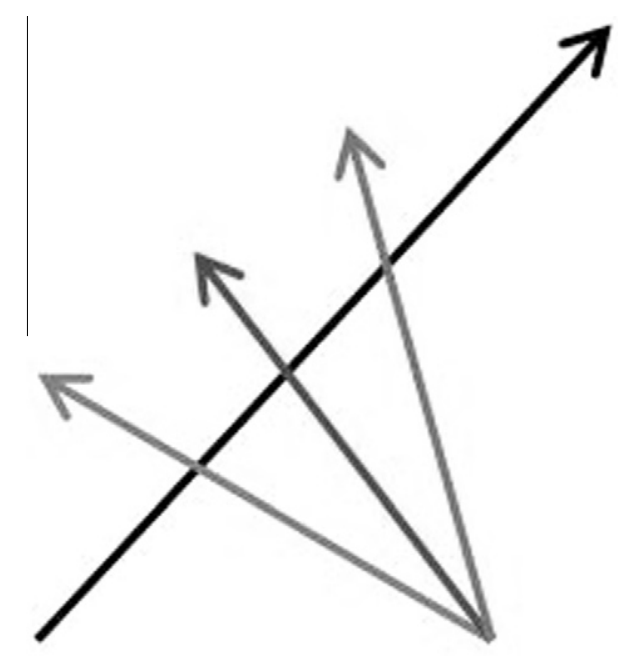

Figure 7. Differentiating individuals alike in one property.

have no length, only direction, we will require transitions from all other points to completely distinguish the individual from all the others falling under the predicate. Two individuals alike for one property will differ for others, see Figure 7.

Each transition from each zero gives the point another quality, another property, until the sum total serves to characterize the point completely from all other points of view. Two points are identical if they share all of the same transitions from all the same zeroes. Assume two points share all transitions but are non identical. Set one of them as zero and define a transition to the other point. This is a transition one has that the other does not, thus they do not share all transitions.

My preferred philosophical interpretation of this primitive structure of points is a manifold of point-events, or individualized event particulars (Russell, 1926, 1954). Following an up to date taxonomy of events (such as Varzi, 1996) event particulars are individual, concrete occurrences, not repeating types or universals. John's stroll, for example, is different each time he takes it, not a repeating event type occurring each day he strolls. Each quality is a trope, an individualized property existing only in particular instantiations or events, while still covering many different individual events as a type. Free qualities indicate these sorts of properties independent of position. The variety of transitions indicate the concrete qualities of the particular event. And, like Riemann's modes, these properties depend on one another at points where they occur together bound in the individual event or occurrence, but are otherwise considered independent.

\section{Raising Extension: The Exterior Product}

Grassmann's achievement was to explain how to raise extension from an abstract manifold of properties and classification, to a real extended space of measurable, extended invariants, such as objects and realistic systems of objects. The key is the sheer primitiveness of the exterior product, which, like its dual the regressive product, requires neither coordinate systems nor a metric for its definition. The formal properties of the exterior product are defined as follows (following Browne 2009): For elements of grades $\mathrm{m}, \mathrm{k}, \mathrm{r}$ :

1. Associativity: $\left(\alpha_{\mathrm{m}} \wedge \beta_{\mathrm{k}}\right) \wedge \gamma_{\mathrm{r}}=\alpha_{\mathrm{m}} \wedge\left(\beta_{\mathrm{k}} \wedge \gamma_{\mathrm{r}}\right)$

2. Identity: $\alpha_{\mathrm{m}} \wedge 1=\alpha_{\mathrm{m}}$

3. Anticommutivity: $\alpha_{\mathrm{m}} \wedge \beta_{\mathrm{k}}=(-1)^{m k} \beta_{\mathrm{k}} \wedge \alpha_{\mathrm{m}}$ (anticommutative if both elements are of odd grade $)^{6}$

4. Distributivity over addition: $\left(\alpha_{\mathrm{m}}+\beta_{\mathrm{k}}\right) \wedge \gamma_{\mathrm{r}}=\alpha_{\mathrm{m}} \wedge \gamma_{\mathrm{r}}+\beta_{\mathrm{k}} \wedge \gamma_{\mathrm{r}}$

5. Scalar multiplication: $\left(\mathrm{a} \alpha_{\mathrm{m}}\right) \wedge \beta_{\mathrm{k}}=\alpha_{\mathrm{m}} \wedge\left(a \beta_{\mathrm{k}}\right)=\mathrm{a}\left(\alpha_{\mathrm{m}} \wedge \beta_{\mathrm{k}}\right)^{7}$

Two examples pictured below in Figure 8 are the multiplication of a point $\alpha$ by another point $\beta$ to bound vector $\alpha \wedge \beta$, and the multiplication of free vector $A$ by free vector $B$ to a free bivector:

In the event-property interpretation, we hold the first point $\alpha$ fixed, along with one common property it shares with $\beta$, and change its modes of determination through a range, or spectrum, of its other properties until it matches the endpoint $\beta$. We thus use $\beta$ to operate on $\alpha$ and give the change a direction. This change has the property of being extended because we associate all the points through their common property or mode lying along line $\alpha \beta$, and dissociate them through the range of other properties. We then reproduce and add up all of the points produced by this associative-dissociative process.

In the second case, we hold one free vector fixed and operate on it by sliding it along the other free vector. Grassmann's gloss on this process (1995, pp. 50-51) describes how the whole range or 'system' of determinations made by $A$ is changed through the whole range of determinations of $B$, so that a range of $A$ is associated with each individual value $B$ can take, setting the values of $B$ apart by associating each of them with the whole range of $A$ (see also 1995, p.171). This prevents the creation of any associations between any particular value of $B$ and any particular value of $A$. The

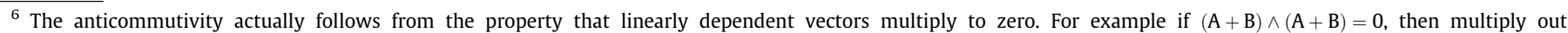
$\mathrm{A} \wedge \mathrm{A}+\mathrm{B} \wedge \mathrm{A}+\mathrm{A} \wedge \mathrm{B}+\mathrm{B} \wedge \mathrm{B}=0$ and set $\mathrm{A} \wedge \mathrm{A}$ and $\mathrm{B} \wedge \mathrm{B}$ also $=0$. Then $\mathrm{B} \wedge \mathrm{A}=-\mathrm{A} \wedge \mathrm{B}$.

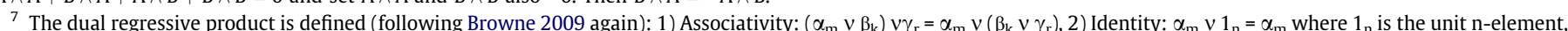

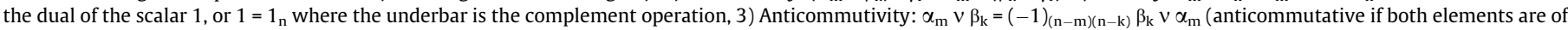
odd grade), 4) Distributive over addition: $\left(\alpha_{\mathrm{m}}+\beta_{\mathrm{k}}\right) v \gamma_{\mathrm{r}}=\alpha_{\mathrm{m}} v \gamma_{\mathrm{r}}+\beta_{\mathrm{k}} v \gamma_{\mathrm{r}}$, 5) Scalar multiplication: $\left(\mathrm{a} \alpha_{\mathrm{m}}\right) v \beta_{\mathrm{k}}=\alpha_{\mathrm{m}} v\left(\mathrm{a} \beta_{\mathrm{k}}\right)=\mathrm{a}\left(\alpha_{\mathrm{m}} v \beta_{\mathrm{k}}\right)$.
} 

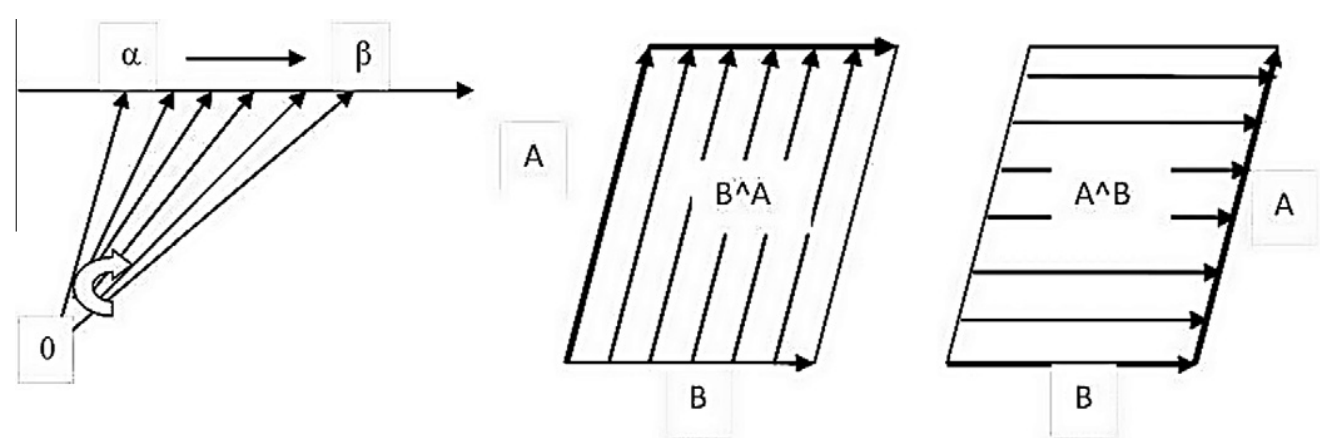

Figure 8. Exterior product of points (Left) and vectors (Right).

similarity with Herbart's crossed-over reproduction series is particularly striking, I find.

There are however some caveats which the idea of a unique tracing process obscures. One, as the algebra reveals in the case of the bivector parallelogram, the area is not of any determinate shape, since these are all equivalent: $A \wedge B=A \wedge(a A+B)=$ $(\mathrm{A}+\mathrm{bB}) \wedge \mathrm{B}$. Also the tracing process itself is indeterminate, since the same area element could have been traced out by another process with a change of basis, so long as it spans the same space. Bringing in the dual regressive product, the area element could equally well be expressed as the intersection of a trivector volume element and a planar element.

Nevertheless, as Grassmann himself points out, the generating process, though indeterminate, does leave two determinate marks on the generated element, the extension itself and its handedness or direction. These marks suggest that some features of a tracing process are indirectly responsible for the extension even if we cannot say exactly which processes they were. Grassmann says "they [the produced extensions] appear as different since they are generated by different evolutions," (1995, p. 50). When we think about a traced extension in space and in time, we imagine sweeping out an area in different ways. In Figure 9 below, imagine a vector $v(t)$ originating from some point, whose tip travels along the curve. The area inside is swept out once, whereas the area below is swept out twice, but in opposite directions, or alternatively, by reversing the parameter $\mathrm{t}$ to $-\mathrm{t}$.

We imagine that opposite handed areas should cancel because we imagine their tracing processes to "undo each other" in time, the one forward the other backward in time. Drawing a left handed figure, like a triangle by connecting its verticies, $\mathrm{ABC}$ is the time reverse of drawing a right handed one CBA. It isn't just a different figure; it is opposite. We do the same thing when we exchange the factors in an exterior product making one the fixed factor and the other the changing, variable factor operating on it. Consider for example the same procedure as $A \wedge B$, but in such a way that we "run alongside" the changing member, $B$, holding it fixed, so that the fixed member A now appears to vary from our point of view. Here we just switch the senses of constancy and variation to reverse the process. With the idea of treating handedness as an actual reversal in the tracing process, making opposite handed extensions opposite and cancelling, it becomes impossible to ignore the fact that extensions really are traced out in some ways rather than others, though not in one determinate way. This is what I take Grassmann to mean by saying different extensions do indicate different methods of evolution or tracing them out.

\section{Connecting Extension with Measurement: A Double Game}

What I believe Grassmann has taught us so far is that the simple, structureless intuition of extension conceals conceptual con-

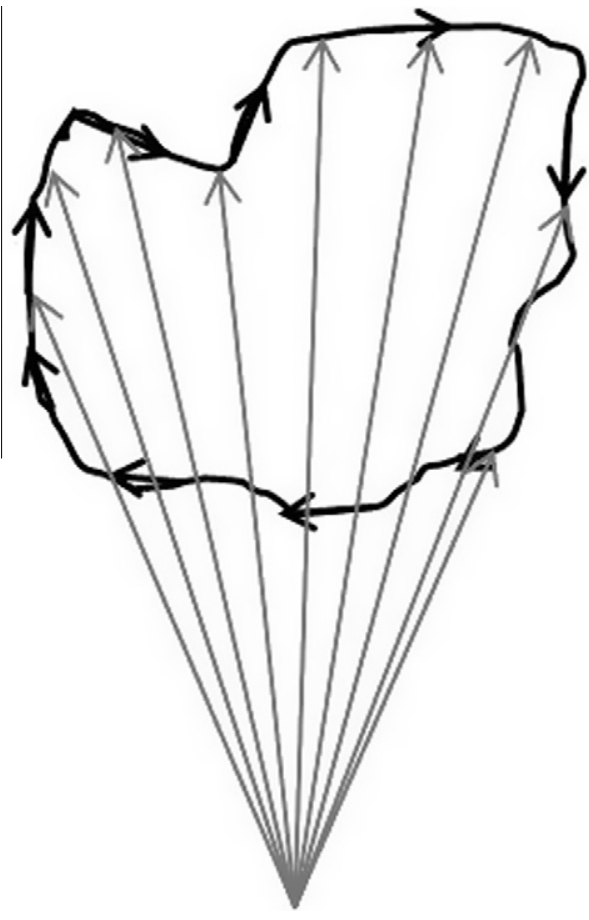

Figure 9. Sweeping out oppositely directed areas.

tent and structure. But the natural objection is still Kant's: given that we always measure extended magnitudes like lengths, areas, volumes, how can any elements prior to that level of extension be measured or experienced? If if they can't, then who cares about them? Clearly all of our direct measurements are of extended magnitudes not unextended ones. So if extension is derived from more primitive ideas, can the concept of measurement also be so derived? Grassmann's algebra is uniquely suited to answer this second question because it is a metric-free algebra, in which the concept of a metric can be probed without just assuming it, just as extension is probed analytically without assuming extension just through considerations of abstract constancy and variation.

What is the connection between the concepts of extension and measurement? Suppose we have experimentally determined a minimum level of extension we can measure, a minimum discrete length or volume element. The level(s) beneath extended measurement, if such there be, consist of entities and lower level extensions, which are not extended for us because they lie beneath the level we can measure. Their extension is abstract or symbolic for us, not concretely intuitible. These lower levels we can deal with symbolically, but if we do imagine them produced by pre-dynamical, pre-causal extension tracing processes, we can only employ an 
analogy with extended dynamical tracing processes and causation familiar to us. The symbolic level is consistent with any and all such possibilities, but also non-unique. We will never find the one process that dynamically traced out the minimum extension in space and time. Instead we will find many such candidates for every extension at the unextended level. On the other hand, as Grassmann urges us to consider, many of these symbolic operations are highly suggestive of alternating, associative-dissociative tracing processes, as if pre-causal processes of some sort did trace out extended magnitudes in particular ways. That cannot be ignored either. So we can play a double game, with the algebraic operations and symbolic combinations on one side, and their pre-causal interpretation as tracing processes on the other, bearing in mind that if push comes to shove, the symbolic level runs the deepest. This correspondence will be as follows:

\begin{tabular}{|c|c|}
\hline Symbolic Order & Pre-Causal Interpretation \\
\hline Free Vector & Property, Mode \\
\hline Point (bound entities) & Dependent Modes, Event \\
\hline $\begin{array}{l}\text { Expanse (free } \\
\text { entities) }\end{array}$ & Independent Modes \\
\hline Exterior Product $\wedge$ & $\begin{array}{l}\text { Extension Tracing Process or Nexus, } \\
\text { INUS Conditions }\end{array}$ \\
\hline Regressive Product $\vee$ & $\begin{array}{l}\text { Mill's Method of Agreement, Common } \\
\text { Factor }\end{array}$ \\
\hline $\begin{array}{l}\text { Orthogonal } \\
\text { Complement__ } \\
\text { Inner Product } \bigcirc \\
\text { Metric Tensor } \mathbf{g}\end{array}$ & Method of Variations \\
\hline
\end{tabular}

The exterior product multiplies independent elements to an extension; if one element is a multiple of the other the product is zero, $A \wedge a A=0$. Consider the product of $A \wedge B \wedge C$ to $D$. We can see $D$ as a causal nexus produced by the independent factors $A, B, C$ and their pre-causal relations. If we hold D constant, we can vary these factors with each other, holding one of them constant and varying the other two. If we hold the other two factors constant, we can vary each factor with the contribution it makes to D. The Method of Variations is just a systematic set of all such relations of constancy and variation among the factors, which we can imagine as performed simultaneously and chained together reciprocally in a system of relations.

The exterior product also has features of INUS conditions, that is, A,B,C are each (I)nsufficient, independent ((N)on-redundant) and (U)nnecessary, but jointly (S)ufficient, constituents of the causal nexus D (Mackie 1980). The factors A,B,C are automatically independent or else $D=0$; they are unnecessary because $D$ need not occur through $A \wedge B \wedge C$, but any other set of sufficient factors that span the space. And $A, B, C$ are each individually insufficient on their own to produce $\mathrm{D}$, but jointly sufficient in combination, as required.

The dual regressive product (v) works when two extensions intersect in a least covering space, or geometrically when their dimensions sum to more than the dimension of the space they are in, so that they are forced to become dependent (Grassmann, 1995, pp. 199-202). Let $\alpha_{m}, \beta_{k}, \gamma_{p}$ be elements with $m+k+p=n$, where $\mathrm{n}$ is the 'least covering space' of them all. Then we can set:

$(\alpha \wedge \gamma) \vee(\beta \wedge \gamma)=(\alpha \wedge \beta \wedge \gamma) \vee(\gamma)$

${ }^{8}(\alpha \wedge \gamma) \vee(\beta \wedge \gamma)=\mathrm{a} 1_{n} \vee(\gamma)$, where $1_{\mathrm{n}}$ is the unit n-element, by duality, the identity element for $\vee$. $(\alpha \wedge \gamma) \vee(\beta \wedge \gamma)=\mathrm{a}(\gamma)$

The regressive product thus isolates the "common factor" from two dependent nexuses, up to a congruence a $(\gamma)$, if there is a "least nexus' where more factors are forced to become dependent and overdetermining. We can compare this symbolic procedure to Mill's method of agreement, where if an effect occurs under a variety of different circumstances, we find the factor common to them all and call it the cause. If three books $\mathrm{X}, \mathrm{Y}, \mathrm{Z}$ are bestsellers produced by author, editor, designer teams $A \wedge B \wedge C, A \wedge D \wedge E$, $A \wedge F \wedge G$, we conclude that the author is the cause, when he works with the others, $(A \wedge B \wedge C) \vee(A \wedge D \wedge E) \vee(A \wedge F \wedge G)=A$, while the particular choice of the others seems not to matter.

I am certainly not suggesting these methods of causal analysis are free of all counterexamples, or even suggesting they analyze the intuitive features of causation. I am merely pointing out a pre-causal interpretation the symbolism seems to support. For example, working with the regressive product, and beginning with a set of higher order extended nexuses, we can think of the lower order elements as isolated out of their occurrences in higher order nexuses, rather than building them up from scratch at the unextended level. This is closer to our actual situation of always dealing with measureable extensions, but both views are equally acceptable interpretations of the symbolism.

\section{The Metric Tensor as the Link Between Extension and Measurement}

These comparisons really become interesting when we introduce a metric tensor. Usually this is done by assuming an inner product first, as an operation which takes elements and their dual complementary co-basis elements to a dimension-independent scalar, a real number. The way this is done in differential geometry is by setting up the desired correspondence and using it to define an entire co-algebra of multilinear forms, such that the inner product of the algebraic elements and their co-elements in the other algebra give a real number. For a given basis, $\mathbf{e}_{\mathrm{i}}$ and co-basis $\mathbf{e}^{\mathrm{j}}$, these inner products of all with all give the matrix components of the metric tensor in that basis: $\mathbf{e}_{\mathrm{i}} \bigcirc \mathbf{e}_{\mathrm{j}}=g_{\mathrm{ij}} ; \mathbf{e}^{\mathrm{i}} \bigcirc \mathbf{e}^{\mathrm{j}}=\mathrm{g}^{\mathrm{ij}} ; \mathbf{e}_{\mathrm{i}} \bigcirc$ $\mathbf{e}^{\mathrm{j}}=\delta_{\mathrm{i}}{ }^{\mathrm{j}}$.

A different approach will be taken here, since the inner product can actually be defined in Grassmann algebra via the more general interior product (see Browne 2009) and is not fundamental, requiring only the metric free exterior and regressive products and the orthogonal complement (which does require the metric for its definition). As with the standard approach, the key is still the definition of the correspondence between basis elements $\mathbf{e}_{\mathrm{i}}$ and their orthogonal complements $\mathbf{e}^{\mathrm{j}}$, which I will symbolize with an underbar $\mathbf{e}_{\text {j. }}$. Following Browne (2009) closely in what follows, consider a particular case, a toy model consisting of a whole system of elements, represented in Figure 10, starting with the scalars at level 0 , then the points and free vectors at level $1\left(0, \mathbf{e}_{1}, \mathbf{e}_{2}, \mathbf{e}_{3}\right)$, then keep on exterior multiplying all basis elements with all to get the elements of the next basis level up, as always counting the odd-permutations of ordered products as negative. Imagine we stop with the bound trivector at level 4 and call this either the highest dimension of the space, or the highest nexus before more factors become dependent.

The magnitude of the trivector basis element is $\sqrt{ }\left|g_{i j}\right|$. It is the basis n-element for this space, because it is the product of all the basis elements, which in geometric terms may be of different lengths and set at different angles, thus not to be confused with the unit $\mathrm{n}$ element $\mathbf{1}_{\mathrm{n}}$ whose measure really is 1 . The general expression that relates these elements is $\left(e_{1} \wedge e_{2} \wedge e_{3} \wedge \cdots \wedge e_{n}\right)=$ 
$\sqrt{ } \mid$ gij $\mid \mathbf{1}_{\mathrm{n}}$. They are only identical in the Euclidean case. If we could calculate the volume of the basis n-element with the inner product, it would be, like any other element, a determinant of a matrix consisting of the inner products of all the basis elements with each other, $\mathbf{e}_{\mathrm{i}} \bigcirc \mathbf{e}_{\mathrm{j}}=\mathrm{g}_{\mathrm{ij}}$, square rooted. Since we are not going to assume the inner product, or a dual co-algebra of multilinear forms, we have to take some other route to the $g_{i j}$.

Imagine then instead that there is some conserved, extended nexus property $\mathrm{V}$ associated with this basis n-element $\mathbf{e}_{1} \wedge \mathbf{e}_{2} \wedge \mathbf{e}_{3}$ under our control and measureable by us, in its own natural units of $\mathbf{1}_{\mathrm{n}}$. Dualing these volume units gives us an exactly parallel scalar measure. Imagine, in Figure 11 below, a "plunger" marked in these units with which we control the volume and hold it fixed. We know that $V=\sqrt{ }\left|g_{i j}\right| \mathbf{1}_{n}$ but we don't know the scalars and must backsolve for them. We attach other "plungers" at all of the basis elements $\mathbf{e}_{1}, \mathbf{e}_{2}, \mathbf{e}_{3}$, each marked only in its own units, such that a unit of $\mathbf{e}_{1}$ does not necessarily correspond to a unit of $\mathbf{e}_{2}$. We also attach plungers to all the co-basis elements marked in their own independent units, $\mathbf{e}_{1}^{*}=\mathbf{e}_{1} \wedge \mathbf{e}_{2}, \mathbf{e}_{2}^{*}=\mathbf{e}_{2} \wedge \mathbf{e}_{3}, \mathbf{e}_{3}^{*}=$ $\mathbf{e}_{1} \wedge \mathbf{e}_{3}$. As independent causal factors, all such units of different basis and co-basis elements are literally of different kinds not to be compared directly. We can mutually relate and constrain them only because we have the constant volume, or better nexuselement, $\mathrm{V}$ to which the elements all multiply, which is a grade and element independent property, allowing for systematic cross-basis comparisons that would otherwise be impossible. ${ }^{9}$

Now we say there must be some orthogonal complement element, $\underline{\mathbf{e}}_{i}$ expressed in the cobasis, $\mathbf{e}_{\mathbf{j}} *$ that is orthogonal to $\mathbf{e}_{\mathbf{i}}$ and equal in measure, so that if $\mathbf{e}_{\mathrm{i}}$ is 1 , so is $\underline{\mathbf{e}}_{\mathrm{i}}: \underline{\mathbf{e}}_{1}=1 / \sqrt{ }\left|g_{\mathrm{ij}}\right|$ $\left(\mathrm{g}_{11} \mathbf{e}_{1} *+\mathrm{g}_{12} \mathbf{e}_{2} *+\mathrm{g}_{13} \mathbf{e}_{3} *\right)$, (see Browne 2009).

In a more familiar notation we can then write $\mathbf{e}_{1}$ as $\mathbf{e}^{1}$, so that $\mathbf{e}_{i}$ $\bigcirc \mathbf{e}^{\mathrm{j}}=\delta_{\mathrm{i}}^{\mathrm{j}}$ eventually, based on the correspondence we are defining here.But this takes things backwards since we don't actually have the inner product until we have defined this correpondence. Our "plunger" problem is to backsolve for the coefficients $g_{\mathrm{ij}}$ of the elements in these equations by the method of variations. We hold the basis n-element $\mathrm{V}$ fixed, and use the plungers to vary the other elements, or dual coelements, with each other in a systematic set of variations, such as we would do when conducting a causal analysis. For example, in this problem we know one constraint already, given that the volume $V=\sqrt{ }\left|g_{i j}\right| 1_{n}$. A symmetric matrix $g_{i j}=g_{j i}$ implies that only $6 \mathrm{~g}_{\mathrm{ij}}$ are unique, and with the constraint that the volume cannot change, we only need to find 5 to solve for the last one.

\subsection{Equations}

$$
\begin{aligned}
1 \mathbf{e}_{1} & =1 \underline{\mathbf{e}}_{1}=1 / \sqrt{\mid g i j} \mid\left(g_{11} \mathbf{e}_{2} \wedge \mathbf{e}_{3}+g_{12} \mathbf{e}_{1} \wedge \mathbf{e}_{3}+g_{13} \mathbf{e}_{1} \wedge \mathbf{e}_{2}\right) \\
1 \mathbf{e}_{2} & =1 \underline{\mathbf{e}}_{2}=1 / \sqrt{\mid g i j} \mid\left(g_{21} \mathbf{e}_{2} \wedge \mathbf{e}_{3}+g_{22} \mathbf{e}_{1} \wedge \mathbf{e}_{3}+g_{23} \mathbf{e}_{1} \wedge \mathbf{e}_{2}\right) \\
1 \mathbf{e}_{3} & =1 \underline{\mathbf{e}}_{3}=1 / \sqrt{\mid g i j} \mid\left(g_{31} \mathbf{e}_{2} \wedge \mathbf{e}_{3}+g_{32} \mathbf{e}_{1} \wedge \mathbf{e}_{3}+g_{33} \mathbf{e}_{1} \wedge \mathbf{e}_{2}\right)
\end{aligned}
$$

\subsection{Method of Variations}

The method of variations is simply to vary certain quantities while holding other quantities fixed. We can construct a table that exhaustively lists all possible variations, setting all of the fixed quantities equal to 1 . Holding $\mathrm{V}$ constant, for example, we vary the units of $1\left(\mathbf{e}_{2} \wedge \mathbf{e}_{3}\right)$ with the units of $1 \mathbf{e}_{1}$, then fixing $1 \mathbf{e}_{1}$ as well we can vary the units of $1 \mathbf{e}_{2}$ with units of $1 \mathbf{e}_{3}$. We divide through these units by $\sqrt{ }\left|g_{i j}\right|$ and bring it outside. Then, exploiting the correspondence we are assuming between the element and its orthogonal complement, we know that the measures of $1 \mathbf{e}_{1}$ and $1 \mathbf{e}_{1}$ are equal and vary $1: 1$. We repeat the whole set of variations for $\mathbf{e}_{2}$ and fore $e_{3}$ and collect up the results of our "plunger experiments," to solve for the 5 unknown $g_{i j}$. This is of course of a local procedure which requires a basis set of elements to experiment in. If these basis elements change, the calculations have to be redone in the new basis. Symbolically, however, we can think of all of these variational cross comparisons being done simultaneously in that basis $\mathrm{g}_{\mathrm{ij}}$ or as the tensor $\mathbf{g}$.

Now we can finally define a true inner product. With: $\mathbf{e}_{i}=\mathbf{e}^{i}: \mathbf{e}_{i}$ $\bigcirc \mathbf{e}_{\mathrm{j}}=\mathrm{g}_{\mathrm{ij}} ; \mathbf{e}^{\mathrm{i}} \bigcirc \mathbf{e}^{\mathrm{j}}=\mathrm{g}^{\mathrm{ij}} ; \mathbf{e}_{\mathrm{i}} \bigcirc \mathbf{e}^{\mathrm{j}}=\delta_{\mathrm{i}}{ }^{\mathrm{j}}$. Example: let $\mathbf{V}=\mathrm{v}^{1} \mathbf{e}_{1}+\mathrm{v}^{2} \mathbf{e}_{2}+\mathrm{v}^{3} \mathbf{e}_{3}$ be the basis representation of a vector; let $\mathbf{V}=v_{1} e^{1}+v_{2} e^{2}+v_{3} e^{3}$ be its cobasis representation. Then $\mathbf{V}_{1} \bigcirc \mathbf{V}^{1}=v_{1} v^{1} \mathbf{e}_{1} e^{1}+v_{2} v^{2} e_{2} e^{2}+v_{3-}$ ${ }^{1}=\mathrm{v}_{1} \mathrm{v}^{1} \mathbf{e}_{1} \mathbf{e}^{1}+\mathrm{v}_{2} \mathrm{~V}^{2} \mathbf{e}_{2} \mathbf{e}^{2}+\mathrm{v}_{3} \mathrm{~V}^{3} \mathbf{e}_{3} \mathbf{e}^{3}=\mathrm{v}_{1} \mathrm{v}^{1}$

$\delta_{1}{ }^{1}+\mathrm{v}_{2} \mathrm{v}^{2} \delta_{2}{ }^{2}+\mathrm{v}_{3} \mathrm{v}^{3} \delta_{3}{ }^{3}=\mathrm{v}_{1} \mathrm{v}^{1}+\mathrm{v}_{2} \mathrm{v}^{2}+\mathrm{v}_{3} \mathrm{v}^{3}=|\mathbf{V}|^{2}$.This inner product definition can then be generalized throughout the algebra to determine the magnitude of all the elements of any grade, given by determinants of inner products of their basis elements.

But bear in mind that the basis elements were only given their measure as combined factors that multiply to the extended basis n-element, which we said we could measure. It is this extended volume or the nexus property, that allows for a concept of element and grade independent measure, and thus ultimately we are only measuring the elements as they participate in $\mathbf{g}$. This was the only extended property we considered accessible to direct measurement. The measures of unextended elements, then, if they have them, are derived indirectly from the extended level. This seems to make serious trouble for the naïve plunger method, which involves variations among the unextended elements, therefore measures, but let's investigate still a little deeper.

Westick with the 4-algebra, whose highest element is a bound trivector, which we shall also consider our metric tensor, i.e., the simplest element we can measure, but also in pre-causal terms the conserved nexus property built of pre-causal relations among the unextended elements to this extension. This property is both the first measureable length, or volume, element and the result of the processes that derive it pre-causally, through associative dissociative tracing processes, at a level prior to extension. The extension is thus built up by the same method of constancy and variation we also use when we analyze it above for purposes of establishing a common measure among its elements.

The unextended elements which multiply to the dimensions of the bound trivector are duals and can be matched with each other in pairs. In our 4-algebra these pairs are the scalar and the bound trivector, the point and free trivector, the bound vector and free bivector, the bound bivector and free vector. These unextended elements can only be measured in these complementary dual pairings, as they participate in producing the extension of the bound trivector element. This extension is like a box with constant volume: if one side gets smaller the others must extend out and we can only measure one extended side at a time. If one element is determined in a more precise range, the other must take on a broader range of dissociated values, because extensions are built up out of such associations and dissociations, with particular values of one element associated with a range of values of another element, a result going all the way back to Herbart's wire argument

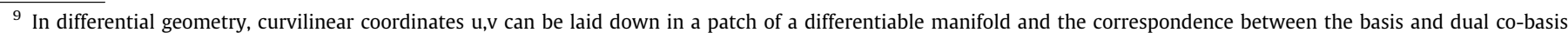

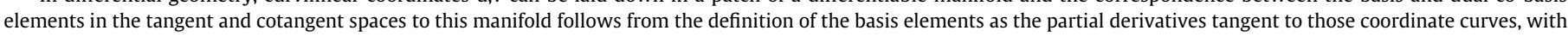

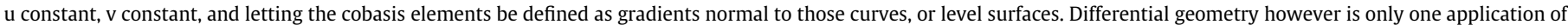

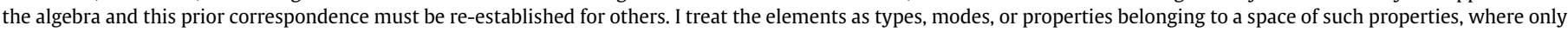
experimental means can decide the question.
} 


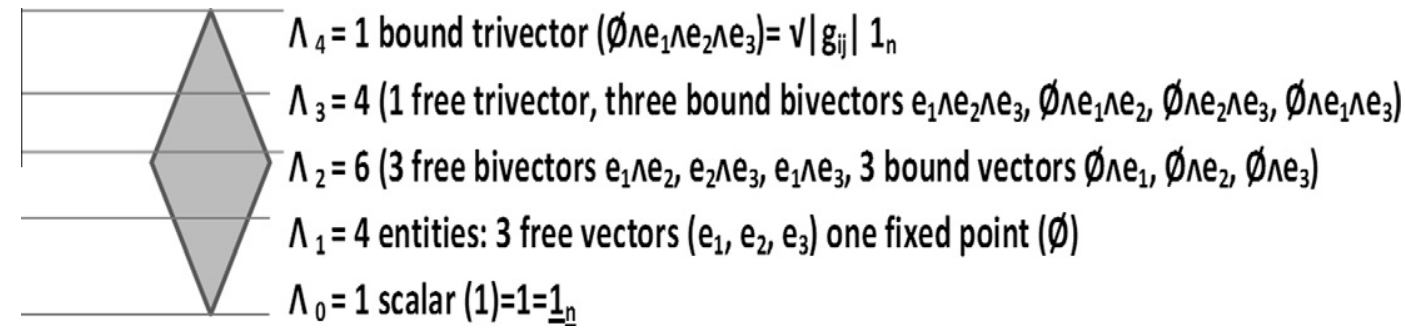

Figure 10. A 4-algebra of bound and free entities.

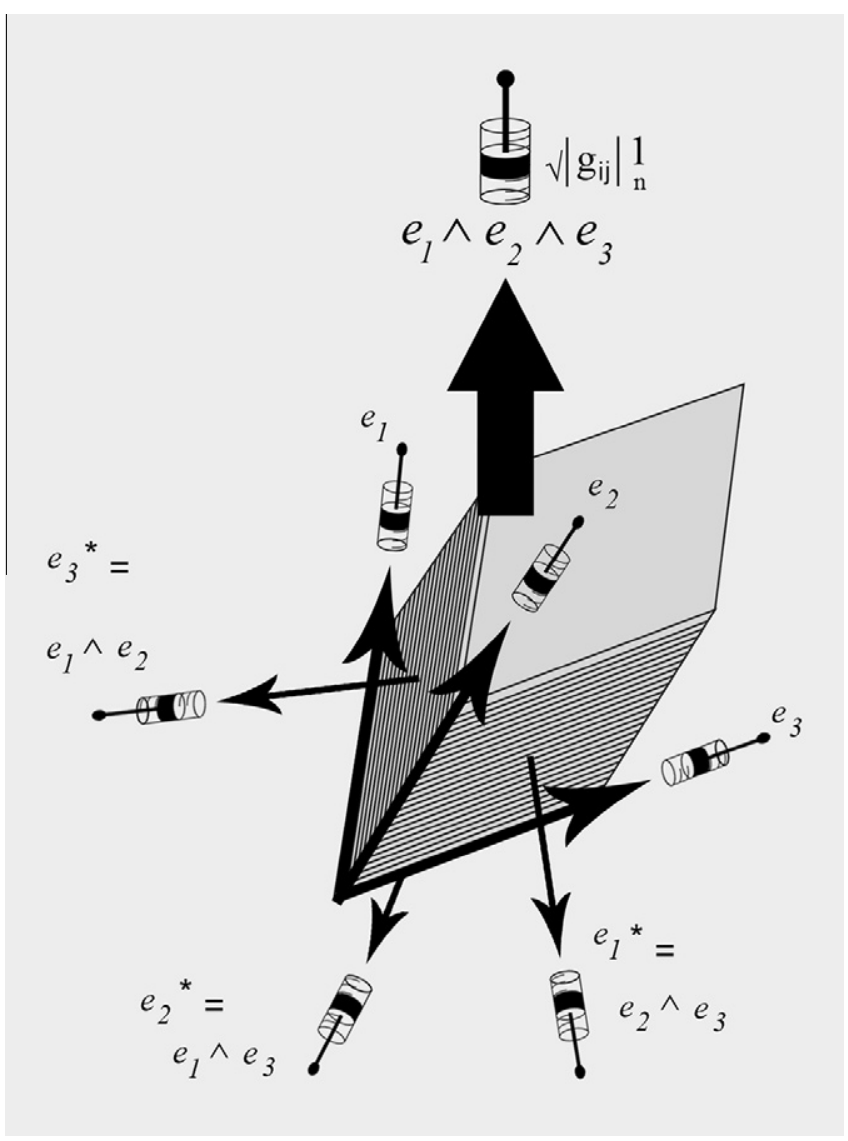

Figure 11. The method of variations with plungers.

and formalized in Grassmann's exterior product. We cannot assign measures to individualized elements beneath the extended level because we can't measure them directly. We can however continue to work our "plunger" method by comparing ranges of values instead of precise ones. When one range narrows, the other expands and this becomes our basis for varying them with each other, not one by one in marks of 1 each.

In our toy model, for example, the consequences are that you can only expect to measure a point event spread over a volume of free space. You can only measure a bound vector (force)spread over a free area. You can only measure a bound bivector (a curl)in a given free direction. It is not true that these unextended elements are unmeasurable; they are rather measureable in ranges determined by their extended combinations with each other. When one range narrows, the other expands, the overall extension remains the same. Thus we can accept both points of view without any conflict: the individual elements do have a measure derived from the extended level, but, and because of this, the measures of individual unextended elements arelimited, because they can only be determined as these elements participate (associatively and dissociatively) in producingmeasurable extension.

\section{Conclusion}

There is so much more to say about this subject, but I think the conclusion is justified that extension is not a structureless intuition but a concept with a well defined structure, which was investigated by Leibniz, Herbart, Riemann and Grassmann, then forgotten, and demoted to the level of the structureless intuition of a 'background drafting board. 'When this extra conceptual structure is uncovered, instead of just assumed, an important connection between the concepts of extension and measurement becomes clear. Grassmann algebra is an explicit analysis of the conceptual structure of extension, but only when we do not geometricize it as extended already, but rather understand Grassmann's work as part of the philosophical tradition that originally began with Leibniz, which does not presuppose extended intuition but rather tries to analyze it further.

\section{Acknowledgements}

This work began during a Fulbright year I spent at the Max Planck Institute for the History of Science in 2004. I would also like to thank Jordi Cat and Amit Hagar for the opportunity to present this paper under the title "Extended Magnitudes" at the Indiana University History and Philosophy of Science Department Colloquium, and for the ensuing discussion. I also thank two anonymous reviewers for the journal and John Browne for his lucid textbook on Grassmann Algebra which was extremely helpful to me. The philosophical interpretations are my own. Special thanks to Sydney Fleming for preparing the diagrams.

\section{References}

Banks, E. (2005). Kant, Herbart and Riemann. Kant-Studien, 96(2), 208-234. Boring, E. (1950) A History of Experimental Psychology.2nd Edition NY: Prentice Hall.

Boscovich, R. (1966, orig. 1763) A Theory of Natural Philosophy. M.I.T. Press. Browne, J. (2009). Grassmann algebra. Melbourne, Australia: Quantica Publishing. Crowe, M. (1967). A history of vector analysis. University of Notre Dame Press. De Risi, V. (2007). Geometry and monadology. Basel: Birkhäuser.

Doran, C., \& Lasenby, A. (2007). Geometric Algebra for Physicists. Cambridge University Press.

Friedman, M. (1998). Kant and the exact sciences. Harvard University Press.

Grassmann H. (1995, orig. 1844, 1862) A New Branch of Mathematics Lloyd Kannenberg, trans. LaSalle, Il: Open Court.

Hatfield, G. (1991). The natural and the normative: Theories of spatial perception from Kant to Helmholtz. Cambridge, MA: MIT press.

Herbart, J. F. (1964). Sämtliche Werke. In Karl Kehrbach \& Otto Flügel (Eds.). Scientia Verlag.

Hestenes, D. (1999). New foundations for classical mechanics. Berlin: Springer.

Kant, I. (2003). Theoretical philosophy 1755-1770. Cambridge University Press.

Lewis, A. (1975). “H. Grassmann's Ausdehnungslehre and Schleiermacher's Dialektik, “. Annals of Science, 34, 103-162.

Mackie, J. L. (1980). The cement of the universe. Oxford University Press.

Petsche, H. J. (Ed.). (2011a). From past to future grassmann's work in context. Basel: Springer. 
Petsche, H. J., Ed. (2011b) Schleiermacher, Fries, Herbart: wer beeinflusste Hermann Grassmann bei der philosophischen Anlagen seiner Ausdehnungslehre von 1844? Manuscript, personal communication.

Riemann, B. (1953 orig 1854). On the hypotheses that lie at the foundation of geometry. In: Gesammelte Werke und Wissenschaftlicher Nachlass. New York: Dover.

Russell, B. (1996, orig. 1897). An essay on the foundations of geometry. London: Routledge.

Russell, B. (1903). The principles of mathematics. Cambridge University Press.

Russell, B. (1926). Our knowledge of the external world (2nd ed.). London: George Allen and Unwin.

Russell, B. (1954 orig. 1927). The analysis of matter. New York: Dover.
Scholz, E. (1982). Herbart's influence on Bernhard Riemann. Historia Mathematica, 9, 413-440.

Schubring, K. (Ed.). (1996). Hermann Günther Grassmann (1809-1877): Visionary mathematician, scientist and neohumanist scholar: papers from a sesquicentennial conference. Dordrecht: Kluwer Academic Publishers.

Stein, H. (1977). Some philosophical prehistory of general relativity. Minnesota studies in the philosophy of science (Vol. 8). Minneapolis: University of Minnesota Press.

Swimmer, A. (1996). The Completion of Grassmann's Natur-Wissenschaftliche Methode (in Schubring op.cit.).

Varzi, A. \& Casati, R. Eds. (1996). Events. Aldershot, England; Brookfield, Vt.: Dartmouth. 